\title{
Structural approach of Ge-X, GeX2 -Ag2X (X=S,Se) glassy systems
}

\author{
P. ARMAND, A. IBANEZ, H. DEXPERT*, D. BITTENCOURT ${ }^{* *}$, D. RAOUX ${ }^{* * *}$ and \\ E. PHILIPPOT
}

Lab. Physicochimie des Matériaux, UM II, 34095 Montpellier Cedex 5, France

*LURE, Bât. 209 D, Universite de Paris Sud, 91405 Orsay, France

** Instituto de Fisica-FEP, Univ. Sao Paolo, C.P. 20516 Sao Paolo, Brazil

${ }^{* * *}$ Lab. Cristallographie, U.P.R. 5031, CNRS, 38042 Grenoble, France

\begin{abstract}
:
This paper presents a summary of our structural results obtained from X-ray Absorption Spectroscopy, Anomalous Wide Angle X-ray Scattering and Small Angle X-ray scattering for germanium chalcogenide glasses. At first, the local and the medium range order existing in the structure of $\mathrm{GeX}_{x}(1.5 \leq \mathrm{x} \leq 9$ and $\mathrm{X}=\mathrm{S}, \mathrm{Se})$ binary glasses have been determined. Then, the results obtained for (1-y) $\mathrm{GeX}_{2}+\mathrm{yAg} 2 \mathrm{X}$ ternary glasses are presented.
\end{abstract}

\section{INTRODUCTION:}

The Ag-Ge-X (X $=\mathrm{S}, \mathrm{Se})$ germanium chalcogenide glasses present an interesting $\mathrm{Ag}^{+}$ionic conduction property at room temperature. This physical property has been well characterized for these glasses in our laboratory [1], but the fundamental mechanisms of the ionic transport process are not yet fully understood from a microscopic point of view. This is partly due to the fact that the structure of these glasses is still not completely established, particularly in the Medium Range Order.(M.R.O). For this reason, a structural approach of these germanium chalcogenide glasses has been undertaken.

All the previous works have demonstrated that germanium sulfide and selenide glasses present important structural analogies. At first, a comprehensive knowledge of $\mathrm{GeXx}(X=S$, Se and $1.5 \leq \mathrm{X} \leq 9)$ binary glasses is essential to be able then to follow the structural changes induced by the addition of a glassy network modifier: $\mathrm{Ag}_{2} \mathrm{X}(\mathrm{X}=\mathrm{S}, \mathrm{Se})$.

An X-ray Absorption Spectroscopy (X.A.S.) study, made at room and low temperatures, has allowed us to determine the local surroundings of germanium, chalcogen and silver atoms in Ge- $X$ binary and $\mathrm{GeX}_{2}-\mathrm{Ag} 2 \mathrm{X}(\mathrm{X}=\mathrm{S}$, Se) ternary glasses. The Short Range Order (S.R.O.) study has been then completed by Anomalous Wide Angle X-ray Scattering (A.W.A.X.S.) measurements which allow us to confirm, first, the XAS results and then to extend M.R.O. characterization to $5-6 \AA$. Two binary GeSes and GeSes glassy compositions have been analysed by AWAXS, the ternary glass study is in progress.

Finally, a more "macroscopic" structural approach has been made by using Small Angle X-ray Scattering (S.A.X.S.) in order to check the homogeneity of the binary and ternary glasses in a range 10 to $1000 \AA$.

In this paper, we present our main structural conclusions for $\mathrm{Ge}-\mathrm{X}$ and $\mathrm{GeX} 2-\mathrm{Ag} 2 \mathrm{X}$ glassy systems obtained from X.A.S., A.W.A.X.S. and S.A.X.S. experiments made at LURE (ORSAY).

\section{2. $\mathrm{GeX}_{\mathrm{X}}(\mathrm{X}=\mathrm{S}, \mathrm{Se})$ GLASS FORMERS:}

\section{a) EXAFS results:}

The EXAFS experiments, made at room temperature, have allowed us to characterize the first coordination spheres of chalcogen and germanium atoms in binary glasses [2-4] using $\alpha \mathrm{GeX} 2$, Ge and $\mathrm{X}=\mathrm{S}$ or Se crystalline phases as structural references. In the whole glassy range, our results are in agreement with the 4-2 coordinated network (fourfold $\mathrm{Ge}$ and twofold X-sites). For (g)GeX2 stoichiometric glass, homopolar bonds have not been found, but when the atomic composition moves off the $\mathrm{GeX}_{2}$ one, the homopolar bond proportion increases: $\mathrm{Ge}-\mathrm{Ge}$ bonds for $\mathrm{Ge}$ enriched glasses $(\mathrm{x}<2)$ 
and $X-X$ bonds for chalcogen enriched compositions $(X>2)$, figure 1. This result supports the idea that the formation of these binary glasses is controiled by a chemically ordered process in agreement with the chemically ordered network model [5].

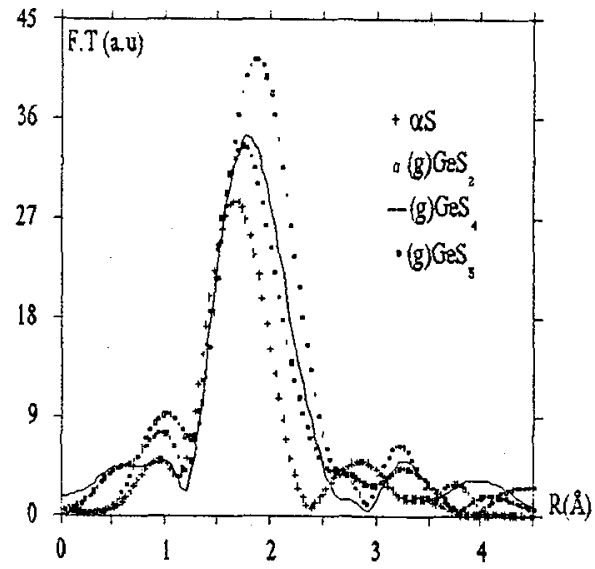

Figure 1: $\mathrm{k}^{2} \times(\mathrm{k})$ Fourier transform moduli, obtained from the $\mathrm{S} \mathrm{K}$-edge EXAFS study for S-enriched GeSx glasses $(x \geq 2)$ compared with the $\alpha S$ one.

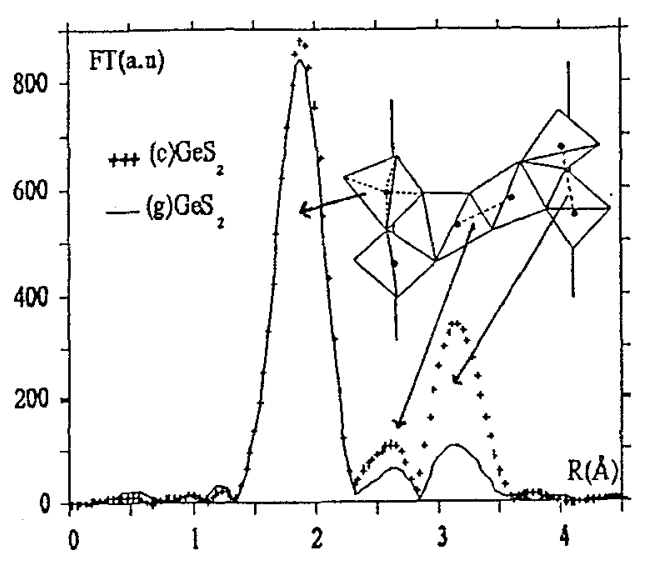

Figure $2: k^{2} \times(k) F . T$. moduli obtained from the $\mathrm{Ge}$ K-edge EXAFS study for (g) GeS2 and aGeS2 which are related to $\alpha \mathrm{GeS} 2$ crystalline structure.

Low temperature Ge K-edge EXAFS experiments have been undertaken to decrease the thermal motion and thus weaken the Debye Waller factor values [6]. From this process, 35K spectra of sulfide glasses have allowed us to characterize the first three Ge coordination shells. It can be demonstrated that the Ge surroundings of $(\mathrm{g}) \mathrm{GeS} 2$ are very similar to those encountered in the crystallized high temperature form $\alpha \mathrm{GeS}_{2}$ [7], taken as structural reference, figure 2 . In $\alpha \mathrm{GeS}_{2}, \mathrm{Ge}$ is located in a regular tetrahedral site $(4 \mathrm{Ge}-\mathrm{S}=2.22 \AA)$. The crystalline structure is built up from [GeS3 $]$ n chains of $\mathrm{GeS}_{4}$ corner sharing tetrahedra, these chains themselves are connected by $\mathrm{GeS} 4$ edge sharing tetrahedra to form layers, figure 2 . Thus, for $(\mathrm{g}) \mathrm{GeS} 2$, the first Ge coordination is constituted by the tetrahedral surrounding of germanium by sulfur. The second shell sphere is due to short Ge-.-Ge interactions of edge sharing tetrahedra (2.92 $\AA$ ) and the third one corresponds to long Ge----Ge interactions of corner-sharing tetrahedra (3.405 $\AA$ ). These results are in accordance with the "outrigger raft" model $[8,9]$ which considers that $(g) \mathrm{GeX} 2(\mathrm{X}=\mathrm{S}, \mathrm{Se})$ structures are mainly constituted by two-dimensional clusters based on GeX2 crystalline network.

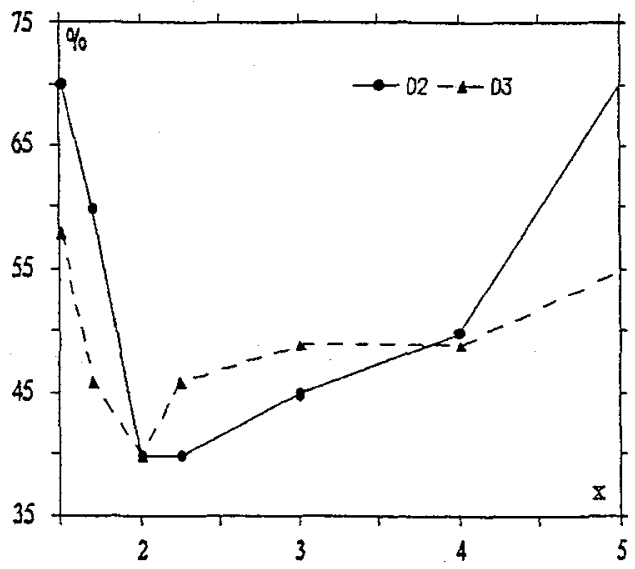

Figure 3:Depolymerization percentage of edge (D2) and corner (D3) sharing GeS4 tetrahedra versus the $\mathrm{GeSx}(1.5 \leq \mathrm{x} \leq 5)$ glassy composition.

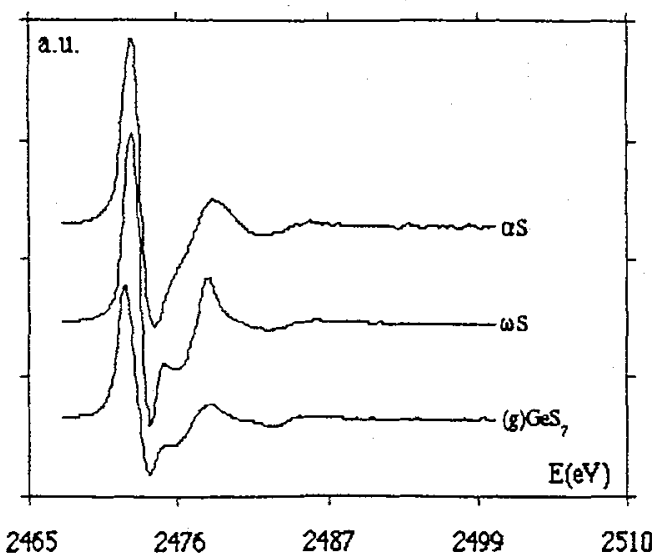

Figure 4: $\alpha \mathrm{S}$ and $\omega \mathrm{S}$ derivative XANES curves obtained at the $\mathrm{S} \mathrm{K}$-edge compared to that of a sulfur enriched binary glass, GeS7. 
In addition, the Ge atom numbers in the glasses, $\mathrm{Ng}(\mathrm{Ge})$ obtained from EXAFS analysis [6] for the second (short Ge...Ge interactions) and the third coordination shells (long Ge...Ge interactions) of the glasses can be used to estimate the degree of depolymerization, $D$, of edge and corner sharing tetrahedra referred to $\alpha \mathrm{GeS} 2$ and to its $\mathrm{Ge}$ atom numbers $\mathrm{N} \alpha(\mathrm{Ge})$. For $(\mathrm{g}) \mathrm{GeS} 2$, these breaking fractions $\mathrm{D}=[\mathrm{N} \alpha(\mathrm{Ge})$ $\mathrm{Ng}(\mathrm{Ge}) 1 / \mathrm{Na}(\mathrm{Ge})$ are both equal to $40 \pm 5 \%$. For all the $\mathrm{GeSx}$ giasses the existence of edge and corner sharing tetrahedra has been shown. Nevertheless, when the atomic composition varies from the GeS2 one, the degree of depolymerization of corner and edge sharing tetrahedra increases significantly inducing an augmentation of the structural distortion in the intermediate range order (3-4A). This increase of tetrahedra depolymerization is certainly due to the high homopolar bond proportion leading to the formation of Ge2S6 units for $\mathrm{Ge}$ enriched glasses or [S]n chains for S enriched compositions. Moreover, the hypothesis of the existence of short [Sln chains or screws rather than S8 rings has been confirmed from S K-edge X-ray Absorption Near Edge Structures (XANES) study [4]. Indeed, the S K-edge XANES spectra of high S content glasses present resonance peaks (due to multiple scattering effects in the M.R.O.) very similar to those of the polymerized $\omega$ sulfur form [10], while the crystalline $\alpha S$ one (S 8 rings) is significantly different, figure 4.

\section{b) AWAXS characterisation:}

In order to complete S.R.O. description, an AWAXS analysis has been made on selenide glasses (sulfur $\mathrm{K}$-edge is not accessible by this technique). The GeSe3 and GeSes compositions have been chosen to follow the structural changes occuring on both sides of the percolation edge suggested to exist around $\mathrm{GeSe}_{4}$ composition. The Differential Distribution Functions of both Ge and Se atoms (DDFGe and DDFse), which specify respectively Ge and Se surroundings, have been extracted for GeSe3 and GeSes glasses (figures 5 and 6).

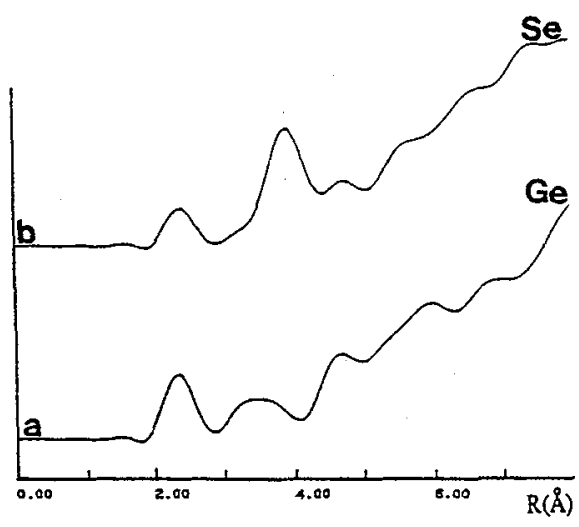

Figure 5: DDFGe (a) and DDFse (b) for GeSe3.

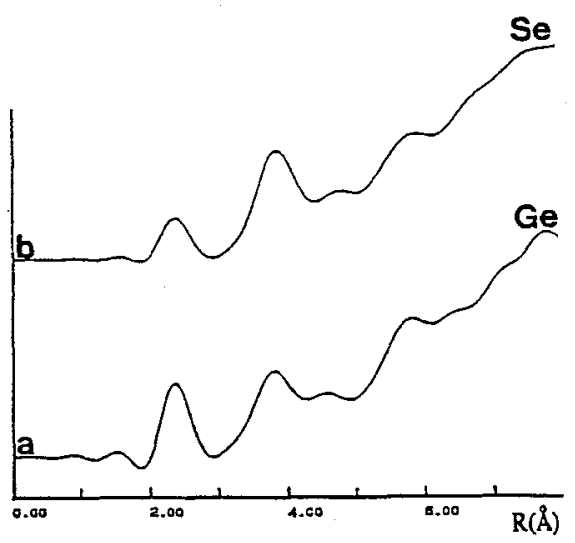

Figure 6: DDFGe (a) and DDFse (b) for GeSes.

These experimental DDFce and DDFse curves, have been fitted until $5 \AA$ using gaussian distribution functions,taking into account the $q$ dependance $(q=4 \pi \sin \theta / \lambda)$ of the ponderation factor. The errors made on the structural parameters obtained from these simulations are around $15-20 \%$ of the coordination numbers, $N$, and about $\pm 0.02 \AA$ for the interaction lengths, $R$.

-For (g)GeSe3, the DDFs curves (figure 5) were modelized using as structural basis the GeSe2 crystalline network which is isomorphic with the $\alpha \mathrm{GeS} 2$ one. Indeed, the first sharp peak of DDFGe (figure 5-a) characterizes the tetrahedral surroundings of germanium by selenium ( $N=3.95, R=2.35 \AA)$. The second extended peak is mainly due to short $(N=0.35, R=3.02 \AA)$ and long $(N=1.6, R=3.52 \AA)$ Ge---Ge interactions of edge and corner sharing GeSe4 tetrahedra. These results and the corresponding Ge-Se-Ge breaking fractions are consistent with the EXAFS results obtained for sulfide glasses. Nevertheless, the addition of Ge---Se interactions $(N=2.7, R=3.45 \AA)$ is necessary to fit correctly this second Ge coordination sphere. The third-neighbour shell, around 4.6 $\mathrm{A}$, is constituted by Ge---Se interactions as for (c)GeSe2. On the other hand, for DDFSe (figure 5-b) Se is twofold coordinated and presents a mixed first-neighbour shell with Se-Ge $(N=1.3, R=2.36 \AA)$ and $\mathrm{Se}-\mathrm{Se}(\mathrm{N}=0.75, \mathrm{R}=2.38 \AA)$ bonds. The second intense peak is induced by Se---Se interactions, around $3.85 \AA$, while the third one is constituted by both Se---Ge and Se---Se 
interactions near to $4.65 \AA$ as for the (c) GeSe2 structure.

-For (g)GeSe5, an important structural change is noted,particularly for the Ge surroundings. For DDFGe,figure 6-a, germanium remains tetrahedrically linked with selenium $(N=4.4, R=2.36 \AA$ ) but the Ge---Ge interactions corresponding to edge and corner sharing tetrahedra are not found. Thus, germanium is only surrounded by Se atoms located respectively around 3.7, 4.2 and $4.9 \AA$. For DDFSe, figure $6 \mathrm{~b}, \mathrm{Se}$ atoms present again a twofold mixed coordination with a more important part of homopolar Se-Se bonds $(N=1.45)$ in this case. Furthermore, the Se---Se interactions near 3.42, 3.83 and $4.72 \AA$ are rather similar to those encountered in the different structural forms of selenium. These results obtained for (g)GeSes seem to demonstrate that this structure is mainly constituted by isolated GeSe4 tetrahedra in an amorphous Se matrix, in agreement with our 35K EXAFS results and with the "cross linked chains model" [11].

\section{c) SAXS experiments:}

All the SAXS spectra recorded for GeSx $(1.5 \leq x \leq 7)$ glasses are very similar. They present a monotonous decay of the scattering intensity $I(q)$ and have been fitted using a Debye Bueche model with correlation lengths of approximately $300 \AA$. These results show that $\mathrm{GeSx}$ binary glasses present electron density inhomogeneities in a length scale inferior to $300 \AA$ in accordance with the formation of different types of clusters in the glassy structure.

After improving our structural knowledge of the binary glasses (especially for ( $g$ )GeX2), a structural study of ternary (1-y) GeX2 + yAg2S glasses has been undertaken.

\section{TERNARY (1-y)GeX2 + yAg2X GLASSES :}

This study has been mainly made on sulfide glasses because the glassy range is more extended for this system. We present here the EXAFS results characterizing the local surroundings of Ge, Ag and S atoms and the first SAXS experiments while an AWAXS investigation of these ternary glasses is in progress.

\section{a) EXAFS resuls:}

As for binary glasses, the three first Ge coordination spheres of ternary glasses have been investigated by $\mathrm{Ge} \mathrm{K}$-edge experiments made at $35 \mathrm{~K}$, figure 7 . The structural parameters resulting from the analytical procedure [6] show an increase of $\mathrm{GeS}_{4}$ tetrahedra distortion when $\mathrm{Ag}_{2} \mathrm{~S}$ is added to (g) $\mathrm{GeS}_{2}$ in accordance with the room temperature results [8]. Furthermore, the $\mathrm{Ag} 2 \mathrm{~S}$ addition seems to induce an important depolymerization of corner and edge sharing tetrahedra and an increase of M.R.O. structural distortion. Even if our data are of very good quality, we are at the technique's limit for second and the third shells of glass compositions containing more than $30 \%$ of silver sulfide $(y=0.3)$, figure 7 . These results can be related to the typical network modifier role played by $\mathrm{Ag}_{2} \mathrm{X}$, leading to the formation of $\mathrm{Ag}-\mathrm{S}$ ionic bonds and the breaking of both double and single Ge-S-Ge bridges.

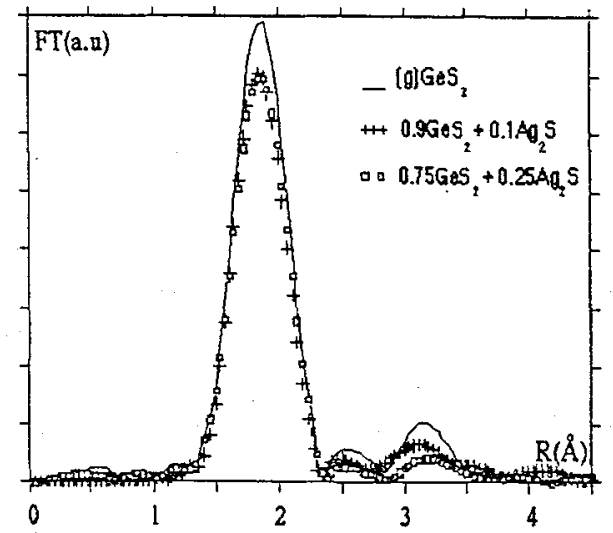

Figure $7: . k^{3} \times(k)$ Fourier transform magnitudes at Ge EXAFS K-edge of $(\mathrm{g})(1-\mathrm{y}) \mathrm{GeS}_{2}+\mathrm{yAg} 2 \mathrm{~S}$ $(y=0.1$ and 0.25$)$ compared to the $(g) \mathrm{GeS} 2$ one.

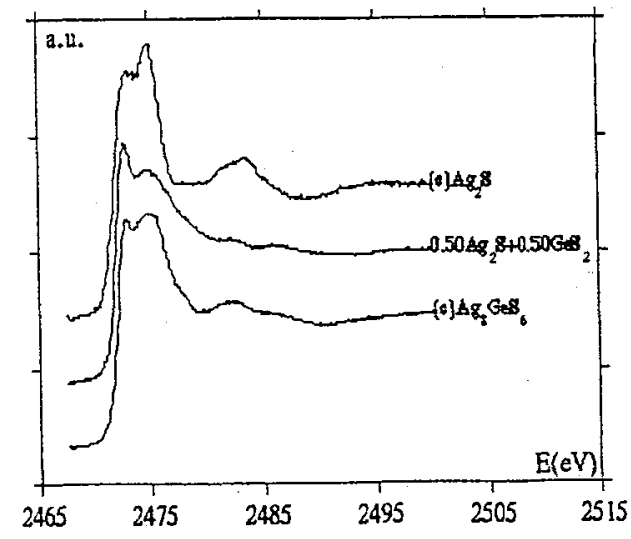

Figure 8: S K-edge XANES spectra of $\beta A g 2 S$, (c) $\mathrm{Ag} 8 \mathrm{GeS} 6$ and (g)0.5GeS2+0.5Ag2S phases. 
Due to the mobility of $\mathrm{Ag}^{+}$cations and to their distorted sites, only the first coordination sphere of silver has been characterized by room and low temperature Ag K-edge EXAFS study. At room temperature, each $\mathrm{Ag}$ atom is bonded to three sulfur atoms $(\mathrm{Ag}-\mathrm{S} \approx 2.51 \AA)$ in very distorted sites. For weak $\mathrm{Ag} 2 \mathrm{~S}$ proportions $(y<0.3)$, the silver sites in the glass seem close to those of $\beta \mathrm{Ag} 2 \mathrm{~S}[12]$ while for high $\mathrm{Ag} 2 \mathrm{~S}$ contents ( $y \geq 0.3)$, the glassy silver sites appear close to those of (c)Ag8GeS6 [13]. These results are consistent with the low temperature EXAFS experiments [14] which show that the Ag thermal behaviour in the glasses $(y \geq 0.3)$ is rather similar to the one of (c)Ag8GeS6 and different to that encountered in $\beta \mathrm{Ag} 2 \mathrm{~S}$ phase. Furthermore, the S K-edge XANES spectra confirm that the glasses with high Ag2S content ( $y \geq 0.3$ ) present sulfur surroundings more similar to those encountered in the Ag8GeS6 crystalline structure, figure 8.

These EXAFS results, obtained for ternary glasses, will be completed by AWAXS investigations already in progress.

\section{b) SAXS experiments:}

Unlike the binary glasses, the SAXS spectra of $(1-y) G e S 2+y A g 2 S$ glasses present an important evolution versus composition, (figure 9):

- For glasses with low Ag2S concentration $(y=0.1)$, the scattering intensity I(q) shows a peak at $q \approx 0.0169 \AA^{-1}$ corresponding to a repetition distance of $370 \AA$ obtained from the Bragg's law. Furthermore, the final part of $I(q)$ obeys Porod's law, $I(q)=f\left(q^{4}\right)[15]$, with a corresponding Porod radius, $R p$, of $55 \AA$.

- When the Ag2S proportion increases, the peak broadenes out to become a shoulder for $y \approx 0.2-0.3$ glassy compositions and disappears for Ag2S enriched glasses ( $y \geq 0.3$ ), figure 9. On the other hand, the Porod region is made worse $\left(\mathrm{I}(\mathrm{q})=\mathrm{f}\left(\mathrm{q}^{-3}\right)\right.$ for $\mathrm{y}=0.2$ and $\mathrm{I}(\mathrm{q})=\mathrm{f}\left(\mathrm{q}^{-2.5}\right)$ for $\left.\mathrm{y}=0.3\right)$ while the corresponding Porod radius is decreased significantly ( $R p=20 \AA$ for $y=0.2$ and $R p=17 \AA$ for $y=0.3$ ). For $y \geq 0.3$ compositions, the scattering intensity, I(q), presents weaker values and a monotonous decay which can be only simulated by a Debye Bueche model.

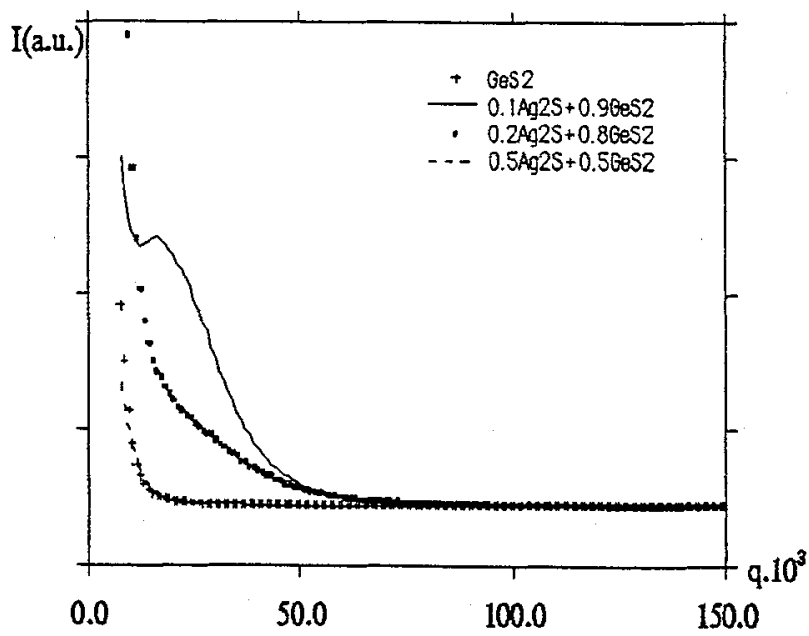

Figure 9: SAXS spectra of (g)GeS2 and (1-y) GeS2+yAg2S ternary glasses.

These results seem to demonstrate that the glassy network modifier, for small amount of Ag2S ,is not well "dilute" in the glassy matrix. This induces the formation of Ag2S clusters with sharp boundary for $y \approx 0.1$ composition. The mean size of these clusters is about $55 \AA$ in this case and their interaction distance is about $370 \AA$. When the Ag2S percentage increases, the Ag2S aggregates become smaller ( $20 \AA$ for $y \approx 0.2)$ and there is no sharp boundary (I(q) deviates from Porod's law). On the other hand, the Ag2S cluster distribution in the glass is loosing order as indicated by the broadening of the peak. This seems to demonstrate that the "dilution" of Ag2S is improved, leading to a more homogeneous repartition of silver in the glass for Ag2S enriched samples ( $y>0.3)$.

This SAXS study, more macroscopic, is consistent with the results obtained for the local structure and would explain particularly the previous results registered for Ag K-edge EXAFS experiments. In order to 
confirm these structural hypothesises, these first SAXS results will be completed by using the anomalous scattering effect (ASAXS).

\section{STRUCTURAL CONCLUSIONS:}

The EXAFS, AWAXS and SAXS analysis presented here are consistent among themselves and suggest for binary glasses that :

-All GeXx binary glasses are chemically ordered and present a 4-2 coordinated network in agreement with the chemically ordered network model [5].

-The structures of glasses near $\mathrm{GeX} 2$ stoichiometry are mainly based on that of the corresponding (c) $\mathrm{GeX} 2$ network with the formation of layered clusters built up from $\mathrm{GeX} 4$ tetrahedral units in agreement with the "outrigger raft" model $[8,9]$.

-When the glassy composition deviates from GeX2 stoichiometry, the homopolar bond proportion and the depolymerization of both corner and edge sharing tetrahedra increases significantly, in accordance with the lowering of glassy transition temperature $\mathrm{Tg}$.

-For chalcogen enriched glasses, over GeX 4 composition, the glassy structure is essentially constituted by isolated GeX4 tetrahedra in an amorphous chalcogen matrix in agreement with the "cross linked chains model" $[11]$.

-The drastic structural evolution registered by AWAXS between GeSe3 and GeSes glasses agrees fairly well with the percolation/demixion threshold hypothesis around GeX4 for these glasses.

For ternary glasses, the $\mathrm{Ag}_{2} \mathrm{~S}$ addition to the $\mathrm{GeS} 2$ glass former gives rise to an important breaking of both double and single Ge-X-Ge bridges and to an increase of the structural distortions of S.R.O and M.R.O. This can be directly related to the typical network modifier role played by $\mathrm{Ag}_{2} \mathrm{X}$.

Nevertheless, for weak $\mathrm{Ag} 2 \mathrm{X}$ proportions, the structure of $(1-\mathrm{y}) \mathrm{GeX} 2+\mathrm{yAg} 2 \mathrm{X}$ glasses $(0<\mathrm{y}<0.3)$ could be demixed and constituted by two cluster types: layered clusters based on the (c)GeX2 network and Ag2X aggregates $(\approx 50 \AA)$ based on the structure of $\beta A g 2 X$. When the Ag2X content increases, its "dilution" is improved leading to smaller clusters $(\approx 20 \AA)$. For high $A g 2 X$ contents $(y \geq 0.3)$ the silver distribution would be more homogeneous in the glass leading to S.R.O.more similar to that of the Ag8GeS6 crystalline phase.

These structural hypothesises for ternary glasses resulting from EXAFS and SAXS experiments, will be completed by the characterization of the M.R.O. by an AWAXS study in progress, in order to better understand the $\mathrm{Ag}^{+}$ionic transport process through the glassy structure.

\section{REPERENCES}

[1] E. Robinel, B. Carette and M. Ribes, J. Non-Cryst. Solids, 57 (1983) 49

[2] C. Peyroutou, S. Peytavin, M. Ribes, H. Dexpert, J. Solid State Chem., 82 (1989) 70; 82 (1989) 78

[3] A. Ibanez, E. Philippot, S. Benazeth and H. Dexpert, J. Non-Cryst. Solids, 127 (1991) 25

[4] P. Armand, A. Ibanez and E. Philippot, submitted to J. Solids State Chem.

[5] G. Lucovsky F. Galeener, R.C.Keeser, R. Geils and H. Six,Phys.Rev.,B10 (1974) 5134

[6] P. Armand, A. Ibanez, H. Dexpert and E. Philippot, J. Non-Cryst. Solids 139 (1992) 137

[7] G. Dittmar and H. Schafer, Acta. Crystallogr. B31 (1975) 2060.

[8] N. Kumagai, J. Shirafuji and Y. Inuishi, J. Phys. Soc. Japan 42 (1977) 1261.

[9] J. E. Griffiths, J. C. Philips, G.P. Espinosa, J. P. Remeika and P. M. Bridenbaugh, Phys. Stat. Sol. (b) $122(1984) \mathrm{k} 11$.

[10] F. Tuinstra, Acta Cryst.20 (1966) 341

[11] J. P. de Neufville, J. Non-Cryst. Solids 8-10 (1972) 85

[12] R. Sadanaga and S. Sueno, Mineral J. 5 (1967) 124

[13] G. Eulenberger, Monatsh. Chem. 108(1977) 901

[14] A. Ibanez, P. Armand,H. Dexpert and E. Philippot submitted to J. Solid State Ionics.

[15] G.Porod, "Small angle X-ray scattering" Academic press (1982) eds O.Glatter and O Kratky. 